University of South Carolina

Scholar Commons

6-15-1986

\title{
Interaction effects among two-dimensional electrons and holes
}

\author{
S. Washburn \\ Richard A. Webb \\ University of South Carolina - Columbia, webbra@mailbox.sc.edu \\ E. E. Mendez \\ L. L. Chang \\ L. Esaki
}

Follow this and additional works at: https://scholarcommons.sc.edu/phys_facpub

Part of the Physics Commons

\section{Publication Info}

Published in Physical Review B, Volume 33, Issue 12, 1986, pages 8848-8850.

Washburn, S., Webb, R.A., Mendez, E.E., Chang, L.L., and Esaki, L. (1986). Interaction effects among twodimensional electrons and holes. Physical Review B, 33(12), 8848-8850. doi: 10.1103/PhysRevB.33.8848 (c) 1986 The American Physical Society. accepted for inclusion in Faculty Publications by an authorized administrator of Scholar Commons. For more information, please contact digres@mailbox.sc.edu. 


\title{
Interaction effects among two-dimensional electrons and holes
}

\author{
S. Washburn, R. A. Webb, E. E. Mendez, L. L. Chang, and L. Esaki \\ IBM Thomas J. Watson Research Laboratory, Yorktown Heights, New York 10598
}

(Received 2 December 1985)

\begin{abstract}
We report large logarithmic corrections to the conductivity of two-dimensional electrons and holes in GaSb-InAs-GaSb double heterostructures. From $\sim 40 \mathrm{mK}$ to $1 \mathrm{~K}$, the conductivity increased with the logarithm of the temperature but with a slope as much as 30 times larger than estimated from the theories of weak localization and carrier interaction. The discrepancy apparently results from electron-hole interactions not included in the theory.
\end{abstract}

The electrical transport of two-dimensional systems is currently under concentrated study by both theorists and experimentalists. ${ }^{1}$ Two-dimensional resistors at low temperatures are not metallic; the conductance decreases logarithmically as the temperature decreases. Two different physical processes cause the two-dimensional conductivity to tend to zero. Weak localization of the individual carriers ${ }^{2}$ (i.e., the coherent backscattering of a single carrier as it diffuses through a random potential) and the Coulomb interactions among the carriers, ${ }^{3,4}$ predict that in zero magnetic field and weak disorder $\left(k_{F} l>1\right)$,

$$
\Delta \sigma=C \frac{e^{2}}{2 \pi^{2} \hbar} \ln (T),
$$

where $C$ is a numerical constant of the order of 1 and $T$ is the temperature of the sample. In the case of localization, the logarithmic slope $C$ is the product of $\alpha$, a scattering parameter, and $P$, the temperature exponent of the inelastic scattering rate. For strictly two-dimensional systems ${ }^{5}$ $0 \leqslant \alpha \leqslant 1$. The prediction for $P$ is model dependent, but for impurity-mediated scattering among the carriers, ${ }^{1} P \simeq 1$ as $T \rightarrow 0$.

The case of repulsive Coulomb interactions among carriers diffusing through a random potential is described by the pioneering calculations of Finkel'shtein, ${ }^{3}$ and independently by Fukuyama and co-workers. ${ }^{4} C$ is given by ${ }^{3,4}$

$$
C=4-3\left(\frac{2+F^{*}}{F^{*}}\right) \ln \left(1+\frac{F^{*}}{2}\right) \text {. }
$$

The parameter $F^{*}$ is equivalent to $F$ (the angular average of the Coulomb interaction) only in the limit when the inverse screening length is much less than the Fermi momentum, i.e., $\kappa<<k_{F}$. In the opposite limit, $F^{*}$ is not calculable.

A magnetic field perpendicular to the plane of the sample tends to enhance the interaction effects and to quench the localization. That is, localization effects are turned off, resulting in negative magnetoresistance, ${ }^{5}$ and interactions yield a positive magnetoresistance. ${ }^{6}$ From analysis of the magnetoresistance associated with the two effects, experimentalists have been able to confirm the presence of both mechanisms in metal-oxide-semiconductor field-effect transistors, ${ }^{7}$ and other two-dimensional systems. ${ }^{8}$

We have performed an experiment to test the effects of the electron-hole (attractive Coulomb) interaction on the quantum corrections to the conductance in weakly disordered samples. To achieve the coexistence of electrons and holes, we employ double heterostructures (grown by molecular-beam epitaxy on $\mathrm{Cr}: \mathrm{GaAs}$ substrates) in which a layer of InAs is sandwiched between two layers of $\mathrm{GaSb}$. The inset in Fig. 1 is a schematic view of the band edges calculated for carriers with $k_{z}=0$ ( $z$ being perpendicular to the plane of the layers).9,10 The conduction band of InAs overlaps the valence band of the GaSb layer. Electrons flow from the $\mathrm{GaSb}$ valence bands into the InAs conduction band, and reside in that two-dimensional well. The holes in the GaSb layer are contained in the triangular wells at the InAs interfaces. In the ideal case, symmetry and charge neutrality imply that there are half as many holes in each of the GaSb layers as there are electrons in the InAs layer. There is, however, experimental evidence both that the wells are not entirely symmetric and that some of the positive charge is trapped at the interface and does not contribute to electrical conduction, at least at high fields. ${ }^{11}$ For all of the samples discussed here, model calculations of the carrier transfer across the heterojunctions indicate that there is only one filled subband in each layer. ${ }^{9}$ If the electron-hole interaction is weak, then the layers of the samples should behave as independent, parallel two-dimensional resistors. ${ }^{12}$

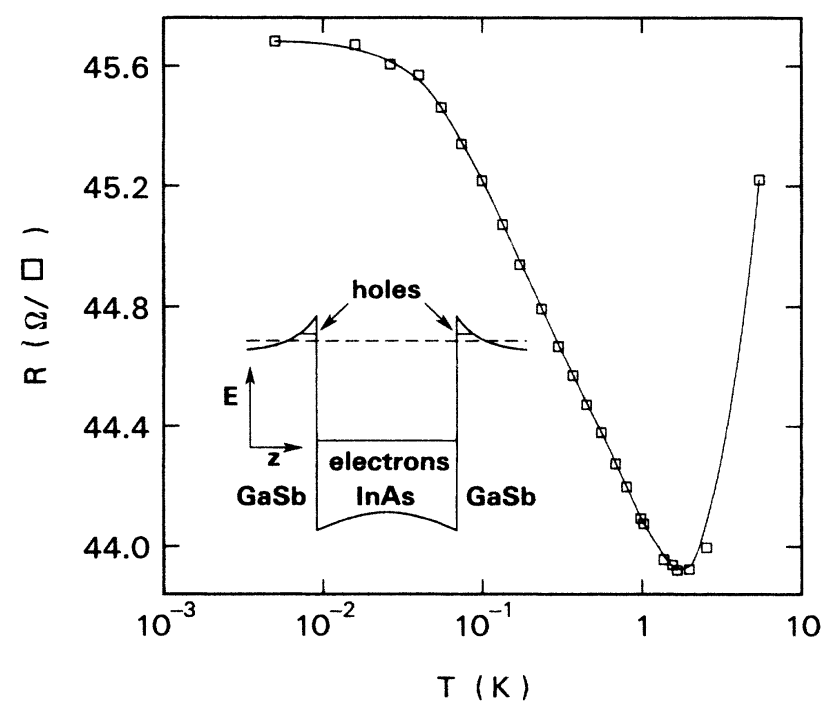

FIG. 1. The sheet resistance of sample II showing the various regions in the temperature dependence. The inset is a schematic of the band structure of the sample viewed on a cross section of the conduction plane; the electron and hole subbands are labeled, and the Fermi energy is indicated by the dashed line. 
Each sample was mounted inside the mixing chamber of a dilution refrigerator. The resistance was measured by a four-probe, ac bridge consisting of a standard resistor, two lock-in amplifiers and a ratio voltmeter. To prevent heating of the electrons, the electric field across the sample was kept to a minimum such that $E \leqslant 1 \mathrm{mV} / \mathrm{m}$. Carrier concentrations and electron mobilities (determined from the Shubnikov-de Haas oscillations) for all of the samples are given in Table I. The hole concentrations can be obtained at high field because the quantized Hall effect reflects only the compensated charge $n_{s}-p_{s} .{ }^{11}$ We have included the compensated carrier concentration (determined for Landaulevel occupation number $i<4$ ) in the table as well.

In Fig. 1 we plot the results of one study of the resistance of sample II as a function of temperature in zero magnetic field. At temperatures greater than a few degrees kelvin, the resistance increases with temperature; resistance ratios for all three samples were typically around 2 . From $T=1$ $\mathrm{K}$ down to $\simeq 40 \mathrm{mK}$, the resistance increased logarithmically as the temperature decreased, and below $30 \mathrm{mK}$, the resistance is constant. For excitations less than $E \simeq 1$ $\mathrm{mV} / \mathrm{m}$, the value of the resistance was independent of excitation, indicating that the saturation at low temperatures was not due to electron heating by the bridge excitation. Such saturation has been observed in a number of experiments. ${ }^{7}$ The saturation might result from spin-orbit scattering, ${ }^{12}$ paramagnetic scattering, ${ }^{5}$ or decoupling of the phonon bath.

Figure 2 shows a typical curve of the conductivity versus temperature in natural units for each of the samples. Assuming that the layers are independent Fermi gases, we expect that the total conductivity is the sum of the layers of the sample in analogy with parallel resistors. The experimental slopes would then be the sum of the quantum corrections from all three layers. It should be noted that holes in $\mathrm{GaSb}$ are about 15 times heavier than electrons in InAs. This implies that the Drude conductivity is dominated by the electron layer.

Negative magnetoresistance from weak localization only occurs when the Landau orbit is larger than the mean free path. Since the mean free paths for the electrons in our samples are so large $(l \geq 1 \mu \mathrm{m})$, the negative magnetoresistance can be present only at fields of $\sim 3 \mathrm{G}$ or less. None of the samples exhibited negative magnetoresistance in this region. For all samples, the magnetoresistance was positive until the onset of Shubnikov-de Haas oscillations. Furthermore, the magnetoresistance near $H=0$ was nearly temperature independent. This is strong evidence that the magnetoresistance is not related to the weak localization or interactions discussed earlier, since these effects have a marked temperature dependence. The temperature-independent positive magnetoresistance results from classical two-band (electron and hole) conductivity; ${ }^{11}$ in other words,

TABLE I. Carrier concentrations and electron mobilities determined from the Shubnikov-de Haas oscillations.

\begin{tabular}{cccccr}
\hline \hline $\begin{array}{c}\text { Sample } \\
\text { No. }\end{array}$ & $\begin{array}{c}n_{s} \\
\left(10^{15} \mathrm{~m}^{-2}\right)\end{array}$ & $\begin{array}{c}n_{s}-p_{s} \\
\left(10^{15} \mathrm{~m}^{-2}\right)\end{array}$ & $\begin{array}{c}\mu \\
\left(\mathrm{m}^{2} / \mathrm{V} \mathrm{sec}\right)\end{array}$ & $k_{F} l$ & $C_{\text {expt }}$ \\
\hline I & 10.7 & 8.4 & 12.4 & 550 & 3.7 \\
II & 9.77 & 8.2 & 14.6 & 588 & 19.8 \\
III & 10.0 & 7.6 & 12.8 & 532 & 59.6 \\
\hline \hline
\end{tabular}

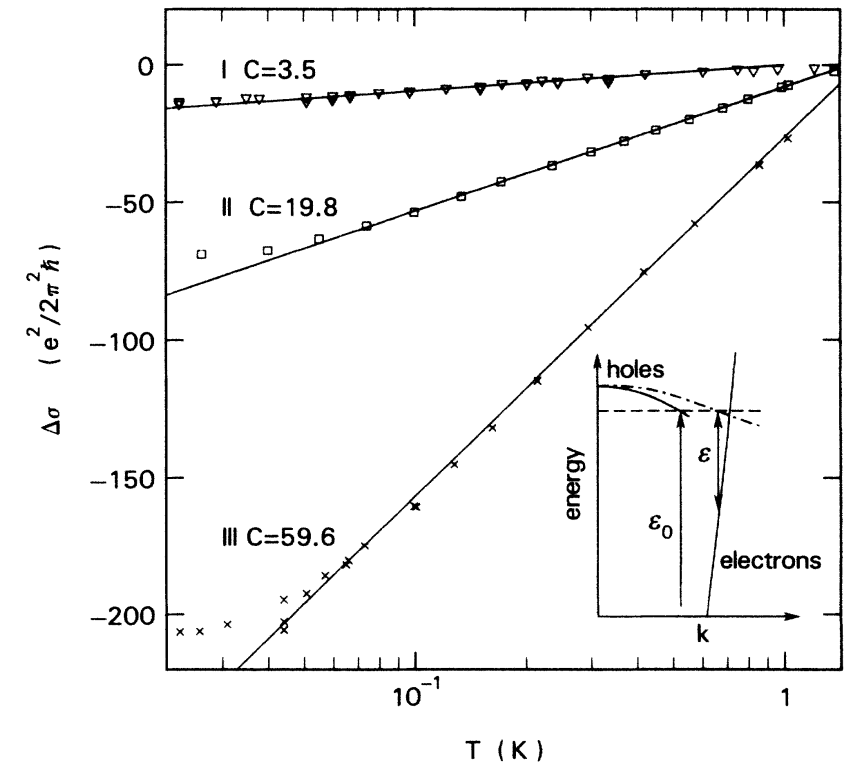

FIG. 2. Representative measurements of conductivity as a function of temperature in the absence of a magnetic field. The slopes $C$ are much larger than the conventional localization and interaction theories predict. The inset schematically illustrates the dispersion for $p_{s}<n_{s}$. The dashed line marks the Fermi level. The gap at the hole Fermi momentum is labeled $\varepsilon_{0}$ for parabolic bands (solid lines) and $\varepsilon$ for a flattened hole band (dot-dashed line).

it is an artifact of the presence of two kinds of carrier. Both weak localization and the Kondo effect ${ }^{13}$ are excluded by the positive magnetoresistance. (The magnetoresistance in parallel magnetic field was also weakly positive, indicating that possible magnetoresistance from Kondo effects was not masked by orbital effects.) It then follows that in all samples, the quantum corrections to the Drude conductivity are dominated by carrier interactions, or by some new mechanism not included in the theory yet.

From the theories of localization and interactions, we expected to find much smaller values of the slope $C$. We approximate the theoretical interaction $F$ in Eq. (2) by Thomas-Fermi screening.,4 Since none of the samples exhibit any negative magnetoresistance, we assume that localization corrections are absent. This leads to (the sum over all three layers) $C=1.7$. In all cases the experimental slope is larger than the theoretical estimate. We do not know how much of this difference might be absorbed by the uncertainty in the screening parameter $F$ or $F^{*}$, but we think that it is unlikely that the interaction theories can be stretched far enough to encompass the experimental slopes (19.8 and 59.6) from samples II and III. Even if we assume that localization contributions from both electrons and holes were masked in the magnetoresistance by some unknown effect, the theoretical estimates are only around $C \simeq 5$-still far too small to explain the results from samples II and III. We are left with the conclusion that, neither the current theories of the Coulomb interactions within each layer of the device, nor the localization theory can account for the huge slopes from samples II and III.

The most plausible explanation for the difference between our experiment and the theory is that the electron-hole interaction is playing an important role in this new system. Implicit in the calculations of Fukuyama and co-workers ${ }^{4}$ is 
the conductivity correction which results from attractive Coulomb interactions. ${ }^{14}$ The particle-particle (Cooper) channel gives rise to the correction

$$
C_{1}=g_{2}-2 g_{4}=\frac{F / 2}{1-(F / 2) \ln \left[E_{F} / \varepsilon\right]} \text {, }
$$

where we have substituted $-F$ for $F$ because the electronhole interaction is attractive. The parameter $\varepsilon$ is an energy scale which gives the typical energy difference between the interacting carriers. In the single-carrier case, this difference is equal to the thermal smearing of the Fermi surface, which is just $k_{B} T$. In InAs-GaSb, the holes and electrons have different Fermi surfaces, and $\varepsilon$ is roughly the separation (conserving crystal momentum) in energy between the two Fermi surfaces. (See the inset in Fig. 2.) The transformation $F \rightarrow-F$ makes the denominator a difference which can approach zero if $\ln \left(E_{F} / \varepsilon\right)$ approaches $2 / F \geq 2$-then $C_{1}$ becomes very large and critically dependent on the carrier concentration (through $E_{F}$ ).

For strictly parabolic dispersion of the carrier energies, the separation between the Fermi surfaces at the hole Fermi momentum is a simple function of the carrier concentrations, $E_{F} / \varepsilon_{0}=\left(1-p_{s} / n_{s}\right)^{-1}$. Empirically, from Shubnikov-de Haas measurements, ${ }^{11}$ one finds $n_{s} \simeq 4 p_{s}$ in these devices. It is assumed that the dormant positive charge exists as ionized interface states. If only the mobile holes contribute to the interactions in Eq. (3), and if the mobility of the holes is independent of magnetic field, then $\ln \left(E_{f} / \varepsilon_{0}\right) \simeq 0.4$. In order to account for the large experimental slopes, this number must be nearer to $2 / F$, implying that the population of mobile holes is nearer to that of the electrons. In light of our experience with these devices, ${ }^{11}$ we think that this is unlikely. It is more likely that the valence band becomes contorted near the heterojunction. There is already theoretical evidence that this occurs in $\mathrm{Al}_{x} \mathrm{Ga}_{1-x} \mathrm{As}-\mathrm{GaAs},{ }^{15}$ where the heavy hole band tends to flatten. If similar flattening occurs in our samples, this would make $\varepsilon$ smaller, and $C_{1}$ larger. How much the trapped holes slow down the electron diffusion is an open question, but it seems likely that they contribute to an even larger slope. The approach of $(F / 2) \ln \left[E_{F} / \varepsilon\right]$ to 1 would account for the very large changes of slope with small changes in carrier concentration and for the large values of the slope.

The original motivation for the experiment was a test of the effects of the electron-hole interaction on the conductivity in weakly disordered samples. Although the evidence from this experiment is not conclusive, it indicates that the interaction between the two species of carriers causes a much more dramatic change in the conductivity than either weak localization or repulsive Coulomb interactions. The only other quantum corrections to the conductivity which are as dramatic, are those which precede the transition to superconductivity. ${ }^{16}$ The difference between the electron pairing in a superconductor and the pairing in the InAs$\mathrm{GaSb}$ system is that in the latter case the conductivity tends to zero because a bound pair (electron plus hole) carries no net charge.

In summary, we have measured the resistances of twodimensional gases of electrons and holes in GaSb-InAs$\mathrm{GaSb}$ quantum wells. In all samples, the logarithmic correction to the conductivity was larger than was expected from either weak localization or interactions among the carriers within each layer. Two of the samples (III and IV) have extremely large corrections. These two slopes are so large that we think that they require a new interaction term in the conductivity. The probable source of the anomalous effects is the electron-hole interaction.

We are particularly grateful to $\mathrm{H}$. Fukuyama for giving us his comments on the data and helping us develop our interpretation of them. We also thank P. A. Lee and S. Maekawa for helpful conversations, and C.-A. Chang, for molecular-beam-epitaxial growth of the samples. This work was partly sponsored by the U.S. Army Research Office.
${ }^{1}$ See the review by P. A. Lee and T. V. Ramakrishnan, Rev. Mod. Phys. 57, 287 (1985).

${ }^{2}$ E. Abrahams, P. W. Anderson, D. C. Licciardello, and T. V. Ramakrishnan, Phys. Rev. Lett. 42, 673 (1979).

${ }^{3}$ A. M. Finkel'shtein, Zh. Eksp. Teor. Fiz. 84, 168 (1983) [Sov. Phys. JETP 57, 97 (1983)].

${ }^{4} \mathrm{H}$. Fukuyama, Y. Isawa, and H. Yasuhara, J. Phys. Soc. Jpn. 52, 16 (1983); Y. Isawa and H. Fukuyama, ibid. 53, 1415 (1984).

${ }^{5}$ S. Hikami, A. I. Larkin, and Y. Nagaoka, Prog. Theor. Phys. 63, 707 (1980); S. Maekawa and H. Fukuyama, J. Phys. Soc. Jpn. 50, 2516 (1981).

${ }^{6}$ P. A. Lee and T. V. Ramakrishnan, Phys. Rev. B 26, 4009 (1982).

${ }^{7}$ D. J. Bishop, D. C. Tsui, and R. C. Dynes, Phys. Rev. Lett. 44, 1153 (1980); D. J. Bishop, R. C. Dynes, and D. C. Tsui, Phys. Rev. B 26, 773 (1982).

${ }^{8}$ See the review by G. Bergmann, Phys. Rep. 107, 1 (1984).
${ }^{9}$ G. Bastard, E. E. Mendez, L. L. Chang, and L. Esaki, J. Vac. Sci. Technol. 21, 531 (1982).

${ }^{10}$ M. Altarelli, Phys. Rev. B 28, 842 (1983).

${ }^{11}$ E. E. Mendez, L. L. Chang, and L. Esaki, Phys. Rev. Lett. 55, 2216 (1985); S. Washburn, R. A. Webb, E. E. Mendez, L. L. Chang, and L. Esaki, Phys. Rev. B 31, 1198 (1985).

${ }^{12}$ S. Washburn, R. A. Webb, E. E. Mendez, L. L. Chang, and L. Esaki, Phys. Rev. B 29, 3752 (1984).

${ }^{13}$ F. J. Ohkawa, H. Fukuyama, and K. Yosida, J. Phys. Soc. Jpn. 53, 2640 (1984), and references therein.

${ }^{14} \mathrm{H}$. Fukuyama and Y. Isawa (unpublished).

${ }^{15}$ T. Ando, J. Phys. Soc. Jpn. 54, 1528 (1985); U. Ekenberg and M. Altarelli, Phys. Rev. B 30, 3569 (1984); 32, 3712 (1985).

${ }^{16}$ A. I. Larkin, Pis'ma Zh. Eksp. Teor. Fiz. 31, 239 (1980) [JETP Lett. 31, 219 (1980)]. 